\title{
Self-assembly of nanoparticle-surfactant complexes with rodlike micelles: a molecular dynamics study
}

\author{
Abhinanden Sambasivam ${ }^{1}$, Ashish. V. Sangwai ${ }^{2}$ and Radhakrishna Sureshkumar ${ }^{1,3, *}$ \\ ${ }^{1}$ Department of Biomedical and Chemical Engineering, Syracuse, NY 13244 \\ ${ }^{2}$ Intel Corporation, Hillsboro, Oregon \\ ${ }^{3}$ Department of Physics, Syracuse University, Syracuse, NY 13244 \\ *Email: rsuresk@syr.edu
}

Composition of all systems studied using MD simulations: Table S1 provides the composition of all systems used to study the effect of NP charge on the formation of the bilayer with respect to the number of molecules of each species.

Table S1: Number of NPs, $\mathrm{CTA}^{+}, \mathrm{Cl}^{-}, \mathrm{Na}^{+}$and Water (W) for different values of surface charge density $(\sigma)$.

\begin{tabular}{|c|c|c|c|c|c|}
\hline $\begin{array}{c}\text { Surface } \\
\text { Charge } \\
\text { Density (б) }\end{array}$ & $\begin{array}{c}\text { Number of } \\
\text { NPs }\end{array}$ & $\begin{array}{c}\text { Number of } \\
\mathbf{C T A}^{+}\end{array}$ & $\begin{array}{c}\text { Number of } \\
\mathbf{C l}^{-}\end{array}$ & $\begin{array}{c}\text { Number of } \\
\mathbf{N a}^{+}\end{array}$ & $\begin{array}{c}\text { Number of } \\
\mathbf{W}\end{array}$ \\
\hline 0.0 & 1 & 324 & 324 & 0 & 19507 \\
\hline-0.33 & 1 & 324 & 324 & 55 & 19452 \\
\hline-0.66 & 1 & 324 & 324 & 107 & 19400 \\
\hline-1.0 & 1 & 324 & 324 & 162 & 19345 \\
\hline-1.5 & 1 & 324 & 324 & 243 & 19264 \\
\hline-2.0 & 1 & 324 & 324 & 324 & 19183 \\
\hline
\end{tabular}



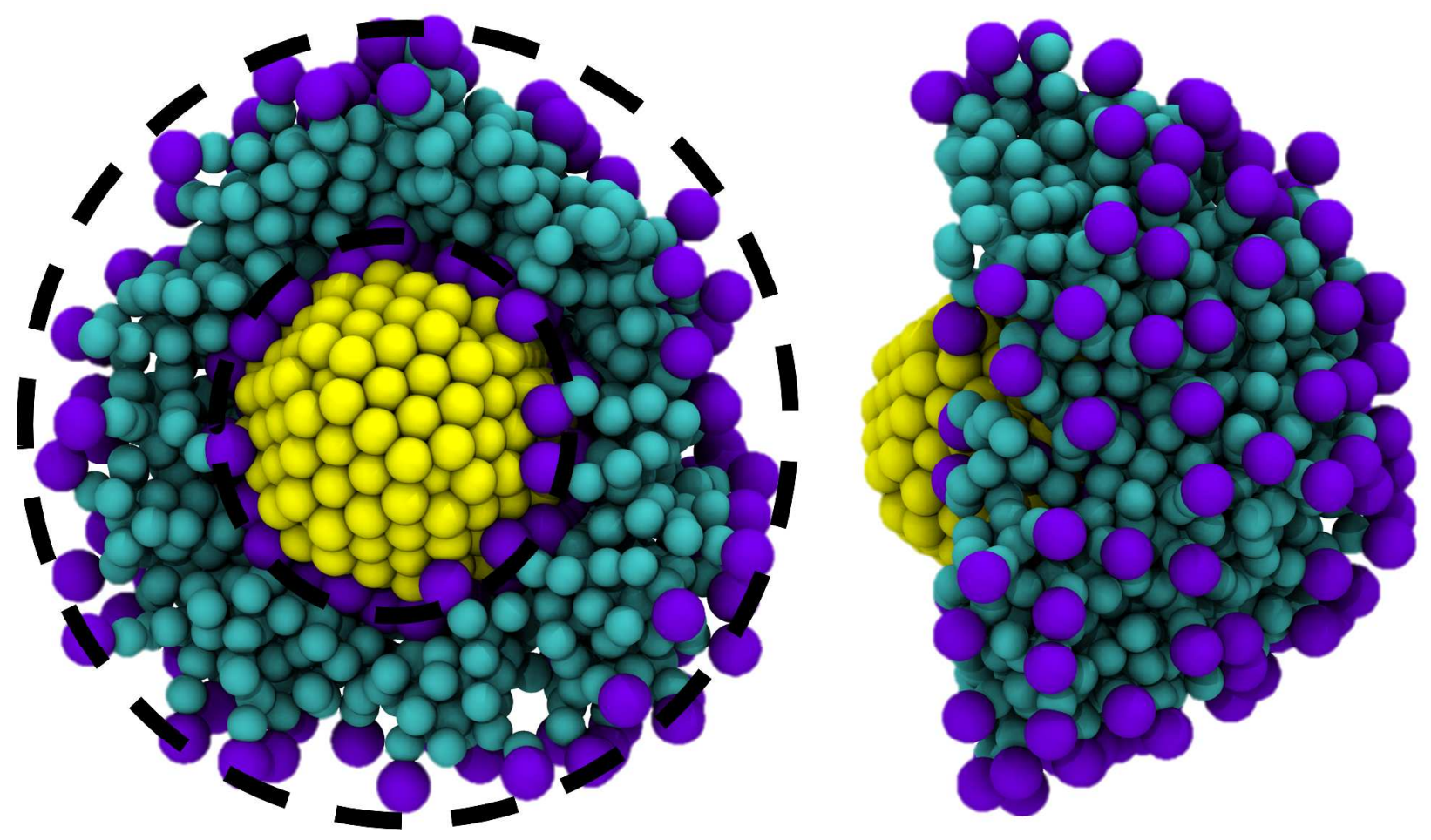

Figure S1: (Left) Snapshot showing a closer look at the vesicular structure. Surfactants that obstruct a direct visualization of the inner layer were removed for clarity. Concentric circles are used to depict the two different layers of the NPSC. (Right) Side view of same snapshot.

Radial distribution of counterions during NPSC formation: Figure S2 shows a plot of the radial distribution function, $\mathrm{g}(\mathrm{r})$, of $\mathrm{Na}^{+}$and $\mathrm{Cl}^{-}$counterions with respect to the center of mass of the NP for $\zeta=-46.75 \mathrm{mV}$. The $\mathrm{g}(\mathrm{r})$ of $\mathrm{Na}+$ ions has a peak at a distance of $2 \mathrm{~nm}$ which shows that $\mathrm{Na}^{+}$ions have a strong affinity to the negatively charged NP surface whereas the peak for $\mathrm{Cl}^{-}$ ions is $\sim 4.5 \mathrm{~nm}$ which is in close proximity to the second layer of head groups in the bilayer. 


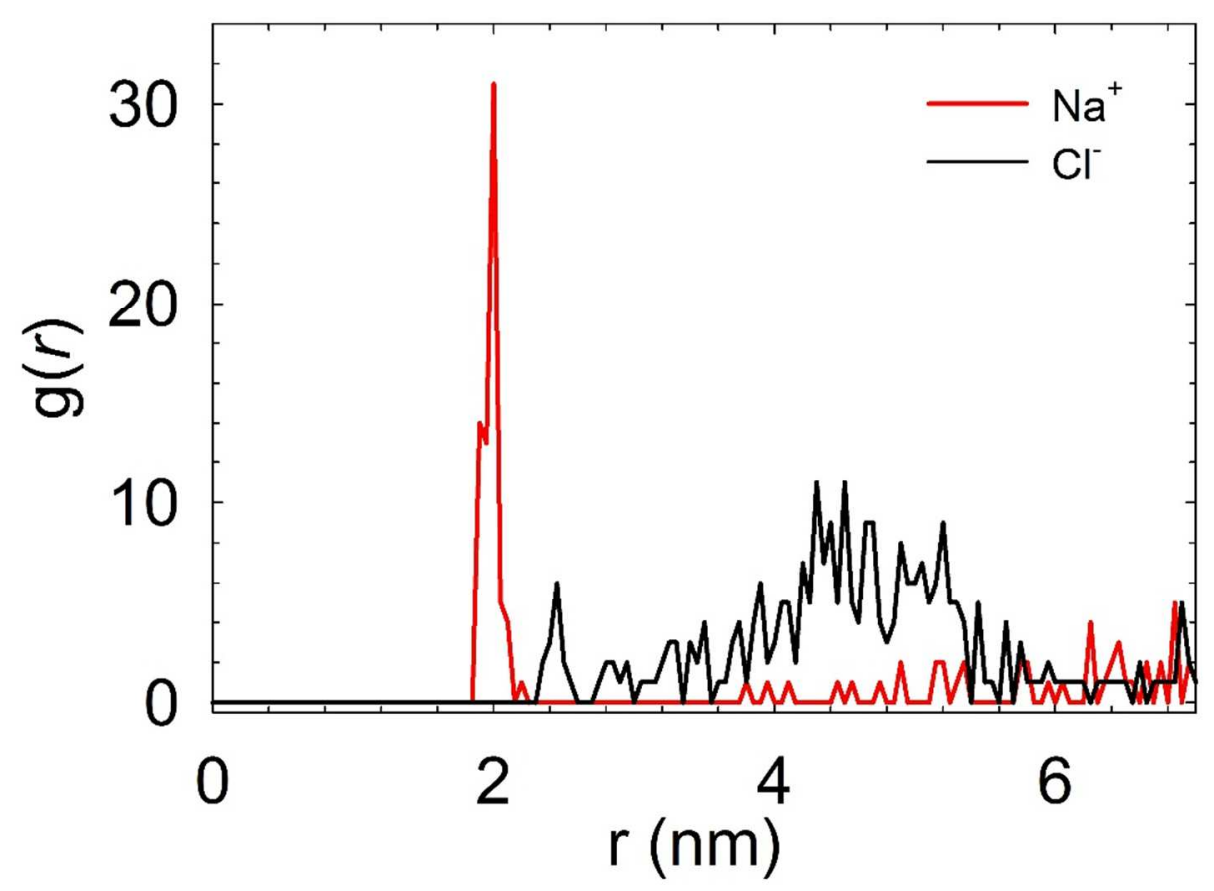

Figure S2: Radial distribution function $\mathrm{g}(\mathrm{r})$ of $\mathrm{Na}+$ and $\mathrm{Cl}$ - ions with respect to the center of mass of the NP for $\zeta=-46.75 \mathrm{mV}$.

Kinetics of surfactant adsorption on nanoparticle (NP) surface: Here we discuss the kinetics of surfactant adsorption on the NP surface for zeta potential $\zeta=-46.75 \mathrm{mV}$ (Fig. S3). It is clearly observed that the inner layer (brown) is formed very rapidly (see inset) because of the strong electrostatic attraction between the surfactant head groups and the oppositely charged NP surface. After the first 10-20 ns, a plateau is observed, suggesting that the surface coverage on the NP is sufficiently large to prevent further surfactant adsorption. However, the outer layer of surfactants (orange) continues to grow because of the presence of free surfactant molecules and small micellar aggregates until the bilayer is saturated. The sudden step increase in the number of surfactants seen is a manifestation of surfactant aggregates associating with the growing bilayer. 


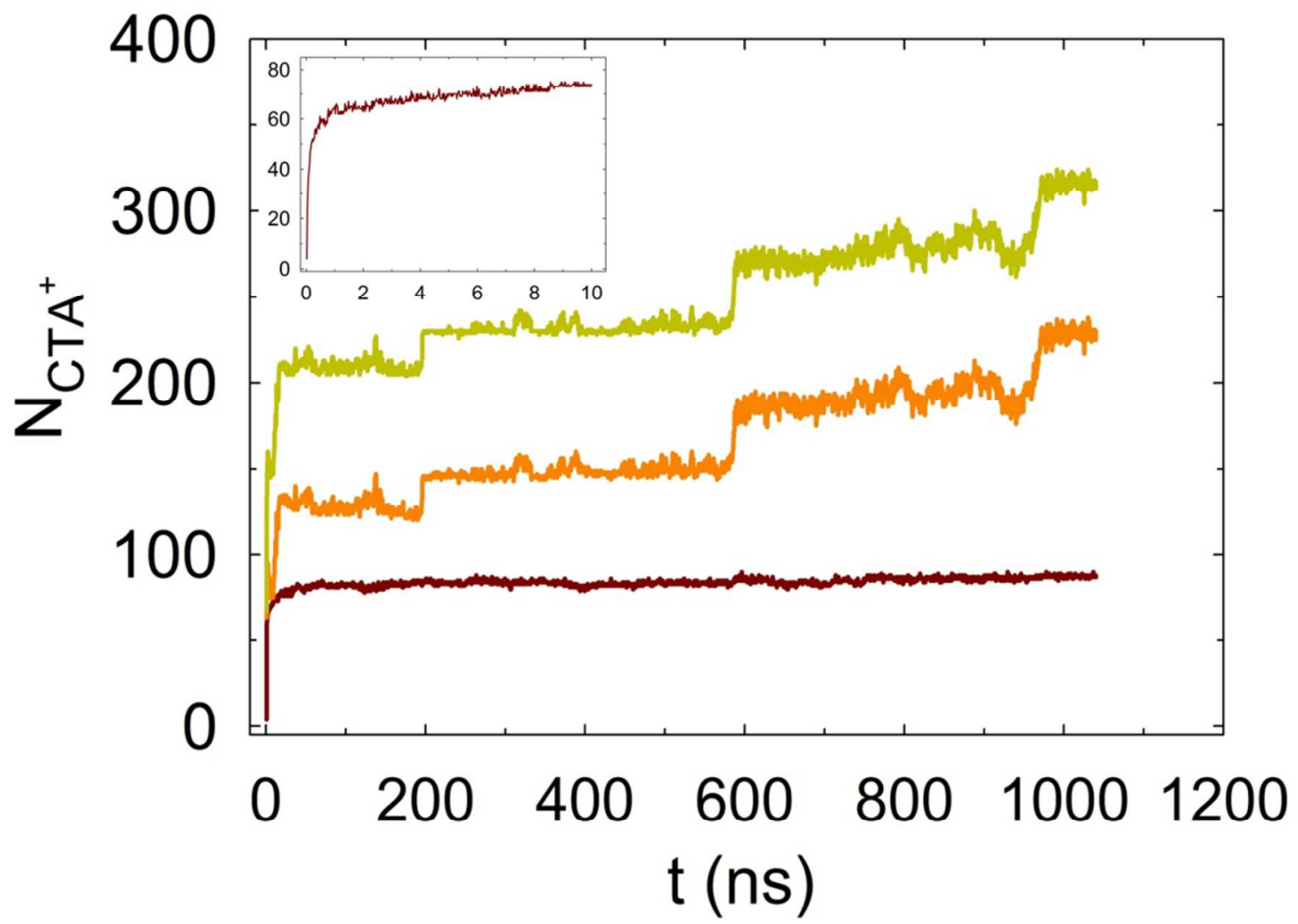

Figure S3: Plot of the number of surfactants adsorbed onto the NP surface vs. time during the formation of the bilayer. The growth of the inner layer (brown), outer layer (orange) and the entire bilayer (green) are all shown separately. The inset shows the growth kinetics of the inner layer during the first $10 \mathrm{~ns}$ of simulation.

Movie 1: Self-assembly of cetyltrimethyl ammonium chloride (CTAC) surfactants and a hydrophobic nanoparticle (NP) leading to the formation of a corona-like monolayer with the surfactant tails adsorbed on to the surface of the NP. The system consists of one NP (yellow) and 324 CTAC surfactants (cyan tails and violet heads) in a box of dimensions $15 \times 15 \times 15 \mathrm{~nm}^{3}$. Water and ions are not shown for clarity.

Movie 2: Self-assembly of CTAC surfactants and a charged metal NP leading to the formation of a vesicle-like bilayer with the cationic surfactant head groups electrostatically attracted to the 
negatively charged surface of the NP. The second layer comprises of surfactants that have their tail groups interdigitated with the tail groups of the first layer and the head groups pointing outward. The system consists of one NP (yellow) and $324 \mathrm{CTA}^{+}$surfactants (cyan tails and violet heads) in a box of dimensions $15 \times 15 \times 15 \mathrm{~nm}^{3}$. Water and ions are not shown for clarity.

Movie 3: Bridging of rodlike micelles with a corona-like NPSC in the presence of sodium salicylate (NaSal) salt. Different color schemes are used for surfactants that are present in the micelle and surfactants that are attached to the NP. CTA ${ }^{+}$in the micelle is represented by pink tail groups and red head groups whereas $\mathrm{CTA}^{+}$in the NP-surfactant complex is represented by cyan tails and violet heads. NP (orange) and Sal' (yellow) are also shown. Water and ions are not shown for clarity.

Movie 4: Bridging of short rodlike micelles with a self-assembled NPSC under the presence of NaSal salt. Same color scheme as movie 3. Water and ions are not shown for clarity. 